\title{
[gw22-e0345] ABERRANT EXPRESSION OF FCГ RIIIA CONTRIBUTES TO DEVELOPMENT OF ATHEROSCLEROSIS
}

Huang Ye ${ }^{1}$, Yin Huijun 1 , Wang Jingshang1', Liu Qian¹, Cao Yali1, Liu Sijin², Chen Keji ${ }^{11}$ Xiyuan Hospital China Academy Of Chinese Medical Sciences, Beijing, China; ${ }^{2}$ State Key Laboratory Of Environment Chemistry And Ecotoxicology Research Center For Eco-environmental Sciences Chinese Academy Of Sciences, Beijing, China

\subsection{6/heartjnl-2011-300867.176}

Purpose Previous studies have shown that Fc receptor III A of immunoglobulin G (FcrRIIIA, also named CD16) was involved in the development of coronary heart disease (CHD). However, the mechanism of CD16 in contribution to CHD development remains largely unclear. Here, we focused on the signaling pathway it stimulates in the initiation and progression of CHD.

Methods We investigated FcyRIIIA mRNA expression in the leukocytes, serum protein level of soluble CD16 (sCD16) and membrane CD16 on monocytes in 100 diagnosed CHD patients and 40 healthy people at similar ages. We determined soluble CD14 (sCD14) to analyse whether monocytes are activated or not. Macrophage colony stimulating factor (M-CSF), TNF $\alpha$ and IL-1 in sera were determined as well. Furthermore, the role of FcrRIIIA in the initiation and progression of CHD was analysed by monocytes adherence to human umbilical vein endothelial cells (HUVECs) in vitro and aortic atherosclerotic formation in ApoE-/-mouse model in vivo.

Results There was a significant increase of Fc $\gamma$ RIIIA at the mRNA level in leukocytes $(1.372 \pm 0.454)$, the protein level of both serum sCD16 (3.237 \pm 0.988$)$ and membrane CD16 on monocytes $(4.8 \pm 2.2 \%)$ in CHD patients when compared to the healthy control. High level of sCD14 in sera $(3.744 \pm 0.896 \mu \mathrm{g} /$ $\mathrm{ml}$ ) was found in CHD patients. Similar to sCD14, the levels of M-CSF $(120.144 \pm 32.123 \mathrm{pg} / \mathrm{ml})$, TNF $\alpha(48.297 \pm 11.545 \mathrm{pg} /$ $\mathrm{ml})$ and IL-1 $(75.842 \pm 20.682 \mathrm{pg} / \mathrm{ml})$ in sera were also higher in CHD patients. Further experiments showed that the elevated level of FcrRIIIA on monocytes dramatically correlated with the adhesive efficiency of HUVECs $(10.5 \pm 5.5 \%)$ in vitro, and also aortic atherosclerotic formation in ApoE-/- mouse model, along with increased levels of TNF $\alpha(41.758 \pm 9.776 \mathrm{pg} / \mathrm{ml})$, IL-1 (71.752 $\pm 13.201 \mathrm{pg} / \mathrm{ml}$;) and soluble E-selectin (sE-selectin) $(2.977 \pm 0.560 \mu \mathrm{g} / \mathrm{ml})$ in sera and both the mRNA level $(3.968 \pm 0.284)$ and the protein level $(0.455 \pm 0.018)$ of MMP-9 in aorta. Additionally, similar to Simvastatin, IVIG pretreatment inhibited this response in $\mathrm{ApoE}^{-/}$mice. 
Conclusion Taken together, these results demonstrate that FcrRIIIA plays a crucial role in the development of athersoclerotic formation by targeting inflammatory cytokines and MMPs, and inhibition of Fc $\gamma$ RIIIA or its signaling may represent a promising approach for the prevention and treatment of CHD. 